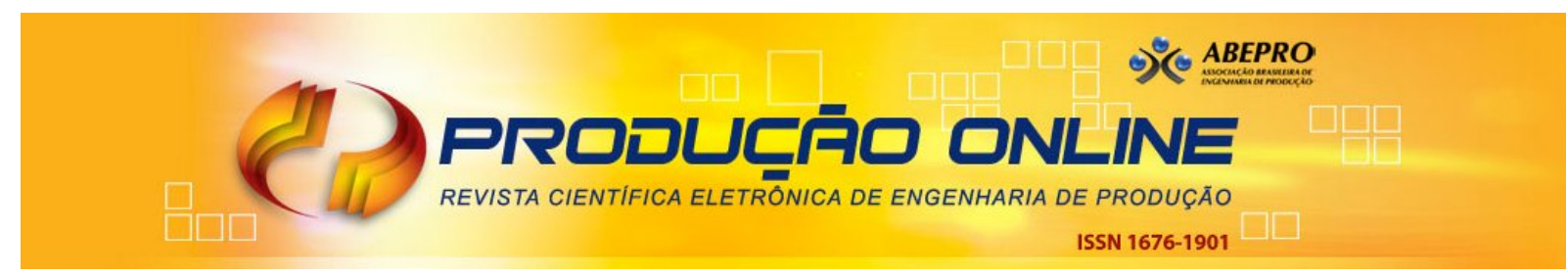

\title{
IMPACTOS DA INDÚSTRIA 4.0 NA ORGANIZAÇÃO DO TRABALHO: UMA REVISÃO SISTEMÁTICA DA LITERATURA
}

\section{IMPACTS OF THE INDUSTRY 4.0 ON WORK ORGANIZATION: A SYSTEMATIC REVIEW OF THE LITERATURE}

\author{
Geraldo Tessarini Junior* E-mail: geraldo.tessarini@gmail.com \\ Patrícia Saltorato** E-mail: saltorato@ufscar.br \\ *Instituto Federal de Educação, Ciência e Tecnologia de São Paulo (IFSP), São Roque, SP, Brasil \\ ** Universidade Federal de São Carlos (UFSCar), Sorocaba, SP, Brasil
}

\begin{abstract}
Resumo: A Indústria 4.0 tem sido caracterizada pela incorporação de emergentes tecnologias de informação ao ambiente de produção, promovendo substanciais ganhos de produtividade e flexibilidade e transformando a natureza do trabalho industrial. Mais do que isso, seus impactos atingem toda a esfera empresarial, política, econômica e social, o que faz com que, não por acaso, venha sendo taxada como a quarta revolução industrial. Nessa perspectiva, este artigo tem como objetivo compreender e apresentar as características, potencialidades e desafios da Indústria 4.0, visando a analisar suas possíveis implicações para a organização do trabalho. Para isso, foi realizada uma revisão de literatura aplicando-se a técnica de revisão sistemática. Os resultados verificados indicam quatro principais impactos: (1) o aumento do desemprego tecnológico, e em contrapartida a criação de postos de trabalho mais qualificados; (2) a necessidade dos trabalhadores desenvolverem uma série de competências para manter suas condições de empregabilidade; (3) a maior interação entre o homem e a máquina; e (4) transformações nas relações socioprofissionais.
\end{abstract}

Palavras-chave: Indústria 4.0. Organização do Trabalho. Quarta Revolução Industrial. Revisão Sistemática.

Abstract: The Industry 4.0 is being characterized by the incorporation of emerging information technologies into the production environment, promoting substantial gains in productivity and flexibility, and transforming the nature of industrial work. More than that, its impacts reach the entire business, political, economic and social sphere, which means that, not by chance; it is being considered as the fourth industrial revolution. From this perspective, this article aims to understand and present the characteristics, potentialities and challenges of the Industry 4.0 , in order to analyze its possible implications for work organization. In order to do so, a systematic review of the literature was conducted applying the technique of systematic review. The results show four main impacts: (1) the increase in technological unemployment, in contrast to creation of more qualified jobs; (2) the need for workers to develop a range of skills to maintain their employability; (3) a greater interaction between man and machine; and (4) transformation in socio-professional relationships.

Keywords: Industry 4.0. Work Organization. Fourth Industrial Revolution. Systematic Review.

\section{INTRODUÇÃO}

Nas palavras de Klaus Schwab (2016, p. 1) "estamos no início de uma revolução que está mudando fundamentalmente a forma como vivemos, trabalhamos e nos relacionamos um com o outro". Trata-se da Indústria 4.0: um novo modelo de 
produção em que máquinas, ferramentas e processos estarão conectados à internet através de sistemas ciber-físicos, interagindo entre si e com a capacidade de operar, tomar decisões e se corrigir de forma praticamente autônoma.

As transformações associadas ao conceito da Indústria 4.0 apresentam potencial para aumentar a flexibilidade, a velocidade, a produtividade e a qualidade dos processos de produção (BCG, 2015a). Seus impactos, contudo, irão muito além: afetarão a economia, as empresas, os governos, as pessoas e o trabalho. Assim, não é por acaso que o conjunto dessas transformações venha sendo retratado como uma quarta revolução industrial (SCHAWAB, 2016), apesar de, diferentemente das revoluções industriais antecedentes, terem sido assim batizadas somente após sua efetiva incorporação ao tecido industrial.

Desde seu início, em 2011, o tema tem recebido substancial atenção de governantes, empresários, universidades e pesquisadores. Está aberto o caminho para um novo campo de pesquisa, com múltiplas facetas e possibilidades, que tem sido abordado de modo interdisciplinar pelas mais diversas áreas da ciência, como engenharias, administração e computação. Entretanto, observa-se na literatura especializada certa fascinação com as diversas tecnologias financiadoras da Indústria 4.0 e seus ganhos de produtividade, em detrimento aos seus possíveis impactos sociais. Estudos que colocam o homem e o trabalho no centro da discussão ainda são escassos. Mais uma vez, assim como nas revoluções anteriores, o trabalhador parece renegado a condição de mero coadjuvante em meio a um turbilhão de inovações que afetarão sobremaneira a sua vida e o seu emprego.

Nasce, com isso, a necessidade desta pesquisa, cujo objetivo é compreender e apresentar as características, potencialidades e desafios da Indústria 4.0, visando a analisar suas possíveis implicações para a organização do trabalho. Não há a ilusória pretensão de encontrar todas as respostas para o tema, mas sim contribuir para um debate esclarecedor sobre o que de fato seria essa revolução: um movimento impiedoso onde não há espaço para o fator humano ou a oportunidade de construção de uma nova organização do trabalho?

Nesse sentido, o trabalho está estruturado da seguinte maneira: nas seções dois e três apresenta-se o arcabouço teórico necessário para compreender o contexto histórico, as características, os desafios e os impactos da Indústria 4.0. Na seção seguinte discorre-se sobre a metodologia de pesquisa empregada neste estudo, a 
qual se baseia em uma revisão sistemática da literatura (RSL). Na seção cinco é apresentada uma visão panorâmica sobre os trabalhos que integraram o portfólio de análise e, por fim, nas seções seis e sete, apresentam-se, respectivamente, os resultados verificados e as conclusões.

\section{CONTEXTO HISTÓRICO}

Independentemente das circunstâncias, a palavra revolução é invariavelmente associada a mudanças profundas e à ruptura com uma realidade anterior. Karl Marx dizia que as revoluções são a locomotiva da história. E ao longo dos tempos, inúmeras revoluções, desencadeadas principalmente por novas tecnologias e por novas formas de perceber o mundo, provocaram mudanças nos sistemas econômicos e nas estruturas sociais (SCHWAB, 2016).

A Indústria 4.0 vem sendo encarada como a $4^{a}$ Revolução Industrial, pois igualmente às anteriores, a inovação tecnológica é o ponto de partida para romper com velhos paradigmas e remodelar drasticamente os sistemas de produção.

A $1^{\text {a }}$ Revolução Industrial, iniciada na Europa no final do século XVIII, introduziu as facilidades da produção mecânica, sobretudo com a criação da máquina a vapor, tornando obsoleta a manufatura artesanal que vigorava até então. A partir dos anos de 1870, a eletricidade, o surgimento das linhas de montagem e a divisão do trabalho derivada do Taylorismo levaram à $2^{\mathrm{a}}$ Revolução. Já a $3^{\mathrm{a}}$, também conhecida como Revolução Digital, teve início na década de 1970 e foi impulsionada pelo emprego das primeiras tecnologias de informação que desenvolveram, ainda mais, a automação dos meios de produção (KAGERMANN; WAHLSTER; HELBIG, 2013; HERMANN; PENTEK; OTTO, 2015; SCHWAB, 2016).

A revolução que se presencia agora teve início em 2011 quando o governo alemão apresentou na Feira de Hannover uma série de estratégias voltadas à tecnologia capazes de transformar a organização das cadeias de valor globais por meio do surgimento de "fábricas inteligentes" (BUHR, 2017; DRATH; HORCH, 2014; SCHWAB, 2016). Desde então, o interesse acadêmico, científico, empresarial e político sobre o tema tem se expandido rapidamente, muito em função do fato de que pela primeira vez uma revolução industrial está sendo observada antes de se tornar, concretamente, realidade (HERMANN; PENTEK; OTTO, 2015). 
Apesar da origem alemã, o modelo tem se espalhado pelo mundo com a adoção de medidas semelhantes em diversos países. Os EUA, por exemplo, anunciaram em 2011 a Advanced Manufacturing Partership (AMP), que consiste na união entre universidades, indústrias e o governo federal para promover investimentos em tecnologias em ascensão no país e, em 2014, sua sucessora, a Accelerating US Advanced Manufacturing (AMP 2.0), trazendo uma série de ações adicionais que deveriam ser adotadas para alavancar a capacidade de manufatura avançada do país. Também se destacam ações em andamento na China, que apresentou em 2015 o Made in China 2025, um programa estratégico para atualizar a indústria no país com diversas metas estabelecidas para 2020 e 2025 e na Coréia do Sul, onde foi criado o Korea Advanced Manufacturing System (KAMS), projeto que tem como objetivo desenvolver novos processos e tecnologias para o gerenciamento e a integração dos sistemas de produção (CNI, 2016; FIRJAM, 2016).

\section{COMPREENDENDO A INDÚSTRIA 4.0}

A Indústria 4.0 é o produto de uma profusão de tecnologias aplicadas ao ambiente de produção, o que Schwab (2016) nomeia de "megatendências". Entre elas, avultam-se os Cyber-Physical Systems (CPS), a Internet of Things (IOT), a Internet of Services (IoS), veículos autônomos, impressoras 3D, robôs avançados, inteligência artificial, Big Data, nanomateriais e nanosensores (SCHWAB, 2016; CNI, 2016; BCG, 2015a).

A combinação dessas tecnologias, como sugerido pelos alemães, tem potencial para habilitar as chamadas Smart Factories, capazes de fabricar produtos de forma mais eficiente com a comunicação e integração entre máquinas, pessoas e recursos (KAGERMANN; WAHLSTER; HELBIG, 2013). Nessas "fábricas inteligentes" máquinas e insumos "conversam" ao longo das operações fabris, agregando flexibilidade aos processos, que ocorrem de maneira autônoma e integrada (CNI, 2016).

Essencialmente, as Smart Factories envolvem a união e harmonização entre os CPS e o uso da loT e da loS nos processos industriais. Estes são os componenteschaves da Indústria 4.0 (HERMANN; PENTEK; OTTO, 2015) e, ainda que não seja o 
objetivo central desta pesquisa, faz-se necessário compreendê-los, sucintamente, buscando entender essa iminente revolução.

Os denominados CPS são uma nova e promissora série de sistemas colaborativos que incorporam profundamente a capacidade cibernética no mundo real (POOVENDRAN, 2010). No ambiente de manufatura, os CPS compreendem máquinas inteligentes, sistemas de armazenamento e facilidades de produção capazes de trocar informações, desencadear ações e controlar um ao outro de forma autônoma, promovendo melhorias nos processos industriais e no gerenciamento do ciclo de vida dos produtos e da cadeia de suprimentos (KAGERMANN; WAHLSTER; HELBIG, 2013). São aptos, ainda, a gerar respostas instantâneas e fomentar um trabalho flexível e distribuído em múltiplas dimensões de tempo, espaço e conteúdo (BAUER et al, 2015; ROBLEK; MESKO; KRAPEZ, 2016).

O sucesso dos CPS, contudo, depende da loT e da loS, que têm sido vistas como o futuro da internet como conhecemos hoje, o que possibilitará a quaisquer objetos do dia a dia se conectarem à rede mundial de computadores. Estima-se que nos próximos anos haverá mais coisas do que pessoas interagindo na web. Isso alterará radicalmente os sistemas manufatureiros pois permitirá que as máquinas tomem decisões sozinhas e que seu controle seja feito de forma remota, proporcionando maior flexibilidade, confiabilidade e eficiência às operações, além da redução de custos (ROBLEK; MESKO; KRAPEZ, 2016). Da mesma forma, a loS agrega considerável valor à cadeia de suprimentos ao permitir que serviços, sobretudo logísticos, sejam fornecidos de forma integrada e combinada com vários canais e participantes (KAGERMANN; WAHLSTER; HELBIG, 2013; HERMANN; PENTEK; OTTO, 2015).

A potencial grandiosidade da Indústria 4.0 somente pode ser compreendida quando ponderados seus possíveis impactos, que por se tratarem de efeitos futuros, podem ou não ser confirmados a depender da capacidade do movimento em superar desafios que já se mostram presentes. As pesquisas apontam para uma série de desafios e impactos na política, na economia, no setor industrial, nos modelos de negócios e na sociedade como um todo. Pode-se concluir que onde houver a possiblidade da Indústria 4.0 provocar mudanças, obstáculos serão enfrentados. É justamente essa amplitude que a torna uma nova revolução e leva Buhr (2017) a concebê-la não como uma revolução tecnológica, mas econômica, política e social. 
No âmbito econômico, a quarta revolução industrial provocará impactos monumentais em todas as variáveis macroeconômicas, como PIB, investimentos, consumo, emprego, comércio e inflação (SCHWAB, 2016). Para tanto, uma enorme quantidade de investimentos será necessária. Davies (2015) estima que somente a Alemanha deva investir, até 2020, 40 bilhões de euros anualmente no projeto, valor que pode chegar a 140 bilhões anuais em toda a Europa. Já os EUA, segundo Buhr (2017), investirão 1,35 trilhão de dólares na Indústria 4.0 nos próximos 15 anos, totalizando 90 bilhões por ano.

Já no âmbito político, para que possa ser viabilizada, a Indústria 4.0 requer que novas regulamentações sejam aprovadas pela Administração Pública, visando à adaptação, à difusão e à proteção às tecnologias digitais. O grande desafio, todavia, é a atuação, em conjunto, entre governos, iniciativa privada e sociedade civil para criar regras, verificações e balanços que permitam manter a justiça, a competitividade, a equidade, a segurança e a confiabilidade na economia e no Estado (SCHWAB, 2016).

As demandas dos consumidores somadas às novas capacidades produtivas $e$ tecnológicas levarão à criação de novos modelos de negócios e serviços orientados a atender às demandas individuais dos clientes e fornecer soluções para problemas em um contexto caracterizado por redes e cooperação entre parceiros de negócios (BUHR, 2017; SCHWAB, 2016; CNI, 2016; KAGERMANN; WAHLSTER; HELBIG, 2013). Isso exigirá das empresas a ampliação de sua capacidade de se conectarem em cadeias globais de valor e de encurtarem o lançamento de produtos no mercado, além de alterarem a forma como se relacionam com clientes e fornecedores, buscando reduzir custos para manter a competividade (CNI, 2016).

No âmbito indústria, muito mais do que apenas benefícios para o chão de fábrica, a introdução dos CPS ao ambiente produtivo possibilitará incríveis ganhos de produtividade, eficiência e flexibilidade em toda a cadeia produtiva, além de permitir a otimização da tomada de decisão e a rastreabilidade de ponta a ponta do processo (KAGERMANN; WAHLSTER; HELBIG, 2013). O BCG (2015a) estima que somente na Alemanha, onde a Indústria 4.0 encontra-se mais avançada, os ganhos de produtividade irão variar de 15\% a 25\%. A flexibilidade das linhas de produção, por sua vez, viabilizará a customização em massa de produtos, ou seja, a produção de bens personalizados conforme as preferências e necessidades do cliente, com tamanha eficiência que mesmo baixos volumes permitirão altos lucros. Há espaço 
também para melhor integração entre os processos de logística e produção e entre máquinas e pessoas (BCG, 2015a; BUHR, 2017; CNI, 2016; KAGERMANN; WAHLSTER; HELBIG, 2013).

A Indústria 4.0 promete ainda apresentar soluções para alguns dos desafios que a sociedade enfrenta atualmente em áreas como saúde, mobilidade urbana e eficiência enérgica com a implantação de redes elétricas inteligentes (CNI, 2016; KAGERMANN; WAHLSTER; HELBIG, 2013). Ao mesmo tempo, Hecklau (2016) alerta para desafios ambientais resultantes da necessidade de maior eficiência no uso dos escassos recursos naturais, exigindo das empresas a busca por novas soluções sustentáveis ao longo de suas ações e processos.

Contudo, os principais impactos sociais ocorrerão na força de trabalho, na empregabilidade e na necessidade das pessoas aperfeiçoarem suas competências para lidar com todas as novas tecnologias e garantir sua empregabilidade, o que faz dessa maior exigência de qualificação, juntamente com as mudanças demográficas já em curso - como o envelhecimento da população - os maiores desafios sociais a serem superados (SCHWAB, 2016; HECKLAU, 2016). É por isso, que, assim como sugerido por Buhr (2017, p. 10) é "imprescindível que olhemos com mais atenção para esse aspecto, para que seja possível identificar onde estão os riscos, mas também as oportunidades para o progresso e a inovação social".

\section{METODOLOGIA}

Esta pesquisa configura-se em um estudo teórico por meio da aplicação do procedimento técnico de revisão sistemática da literatura (RSL). Tal técnica foi empregada visando identificar, avaliar e interpretar as pesquisas relevantes sobre uma particular questão de pesquisa, utilizando-se de uma sequência metodológica bem definida que permite agregar conhecimento e construir saberes (KIETCHENHAM; CHARTERS, 2007; GREENHALGH, 1997).

Para os propósitos deste estudo, adotou-se o modelo de RSL desenvolvido por Kietchenham e Charters (2007), consistindo em uma sequência de três etapas planejamento, condução e apresentação da revisão - cada qual guardando suas respectivas ações. 
Figura 1 - Etapas da RSL

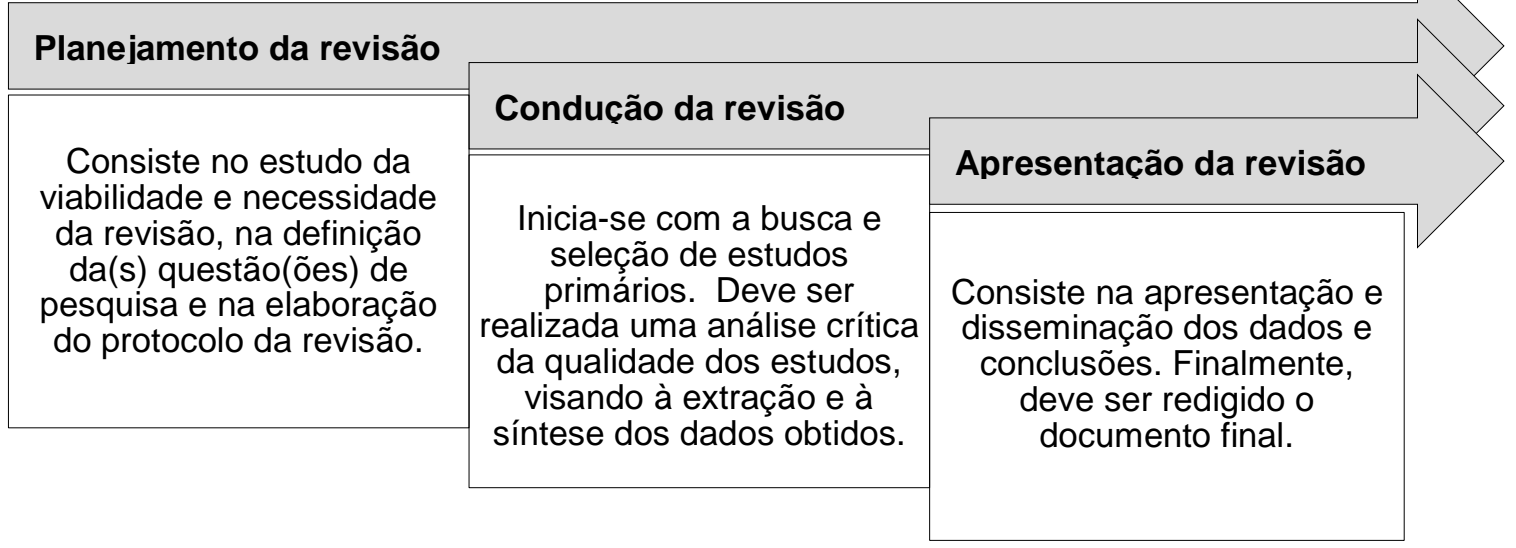

Fonte: Elaborado pelos autores a partir de Kietchenham e Charters (2007)

Iniciou-se a fase de planejamento a partir de uma revisão do escopo, apresentada nas seções 2 e 3, que teve por intuito obter maior familiarização com o tema abordado e a definição do problema e das hipóteses de pesquisa. Nessa fase, também se confirmou a viabilidade e a necessidade desta pesquisa, principalmente a partir dos apontamentos de Kagermann, Wahlster e Helbig (2013), Schwab (2016) e Buhr (2017), cujos trabalhos serviram como embasamento teórico principal. Esta pesquisa também se justifica uma vez que não foi encontrado nenhum estudo nacional semelhante que tivesse como propósito identificar, categorizar e analisar as diferentes percepções apresentadas pelos pesquisadores em relação aos impactos da Indústria 4.0 no trabalho.

Após a realização da revisão do escopo, iniciou-se a revisão sistemática propriamente dita, definindo-se as seguintes questões-problema:

- Quais as projeções feitas pelos pesquisadores em relação às implicações da Indústria 4.0 para a organização do trabalho, de forma geral; e para o trabalhador, de forma específica?

- Considerando a nova dinâmica produtiva projetada pela Indústria 4.0, quais competências e habilidades serão exigidas para que o trabalhador possa manter ou aperfeiçoar suas condições de empregabilidade?

A busca em meio às pesquisas primárias empreendeu-se, em um primeiro momento, de forma automática na base Scopus, escolhida por seu caráter 
interdisciplinar e por ser considerada a maior base de dados referenciais do mundo. Inicialmente, realizou-se uma busca nos títulos, resumos e palavras-chave dos trabalhos disponíveis na base de dados utilizando os construtos "Fourth Industrial Revolution", "Industry 4.0" e "Industrie 4.0". Este último foi adicionado de modo a cobrir possíveis variações linguísticas. Foram recuperados 1.990 documentos. Em seguida, baseados nas questões-problema definidas e na revisão do escopo anteriormente realizada, procedemos à análise detalhada de todas as palavras-chave disponíveis com vistas a identificar as principais keywords associadas ao objeto desta pesquisa e obter o maior número possível de trabalhos correlatos. 10 foram selecionadas e com isso, refizemos a busca nos títulos, resumos e palavras-chave, através da combinação dos construtos demonstrados na figura 2.

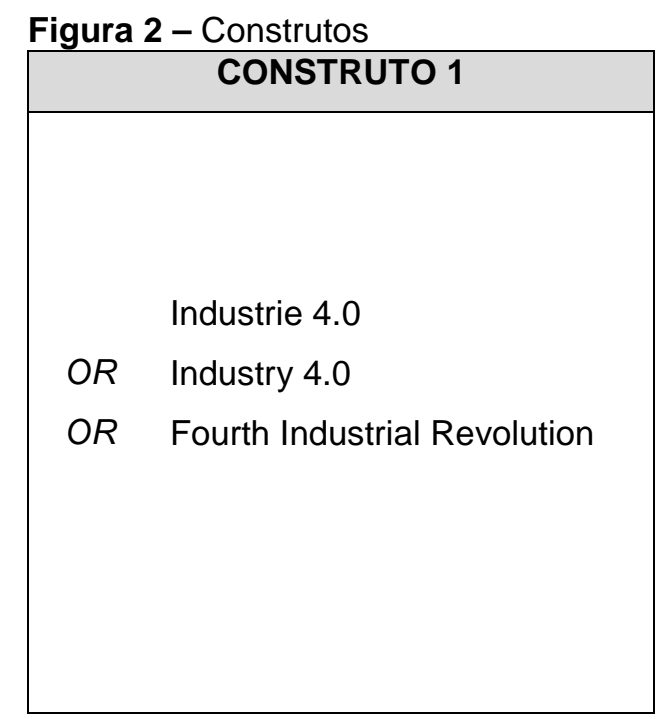

Fonte: Elaborado pelos autores

\begin{tabular}{|ll|}
\hline \multicolumn{1}{|c|}{ CONSTRUTO 2} \\
\hline AND & Digitalization of work \\
OR & Employment \\
OR & Human factors \\
OR & Human machine interaction \\
OR & Qualification \\
OR & Skills \\
OR & Workforce management \\
OR & Work design \\
OR & Working environment \\
\hline
\end{tabular}

Essa busca resultou em 159 documentos. Passou-se à etapa de refinamento filtrando-se somente trabalhos no idioma inglês ou português; publicados em periódicos, conferências ou em capítulos de livros; publicados nas áreas do conhecimento correlatas (Computer Science; Engineering; Decision Sciences; Business, Management and Accounting e Social Sciences) e publicados entre 2011 (ano em que o termo Indústria 4.0 foi utilizado pela primeira vez) e 2017. Restaram 119 trabalhos.

A partir da leitura de todos os títulos e resumos aplicou-se uma nova filtragem onde 81 estudos foram imediatamente excluídos, pois: (1) não focalizavam a Indústria 
4.0 sob a perspectiva do trabalho, apenas tangenciando-o ocasionalmente; ou (2) os artigos completos não estavam disponíveis gratuitamente.

Os artigos remanescentes foram lidos em sua totalidade e para maior segurança no processo de inclusão/exclusão realizou-se a análise do conteúdo de forma crítica, consistindo-se este em um princípio básico da RSL. Nessa etapa, buscou-se, acima de tudo, avaliar três critérios principais: (1) a qualidade dos artigos, tanto teórica como metodológica; (2) a relação com os propósitos desta pesquisa; e (3) o fornecimento de respostas para ao menos uma das questões-problema. Apenas 14 artigos atenderam ao proposto. Sendo esta uma etapa da revisão bastante subjetiva, não está descartada a hipótese de que outros estudos relevantes possam ter sido ignorados e não inclusos na seleção final.

Tendo ainda em vista o pequeno número de trabalhos obtidos a partir da busca automática e a necessidade de expandir o portfólio de análise tencionando eliminar possíveis vieses de pesquisa, foram incorporados ao portfólio reports de renomadas instituições internacionais que parecem despontar como pioneiras na publicação de pesquisas, relatórios e white papers sobre a Indústria 4.0. Entre elas destacam-se o World Economic Forum e o Boston Consulting Group. Ainda que tais documentos não possuam caráter acadêmico, suas contribuições são de altíssimo valor e não poderiam ser desprezadas, pois se tratam da apresentação de dados concretos das primeiras surveys realizadas sobre os impactos no trabalho decorrentes da expansão da Indústria 4.0.

Essa nova rodada de seleção resultou em mais 5 trabalhos, portanto, 19 estudos integraram o portfólio final de análise desta pesquisa e suas conclusões e contribuições são apresentadas nas próximas seções.

Todo esse percurso metodológico é sintetizado no quadro 1. 
Quadro 1 - Percurso metodológico da RSL

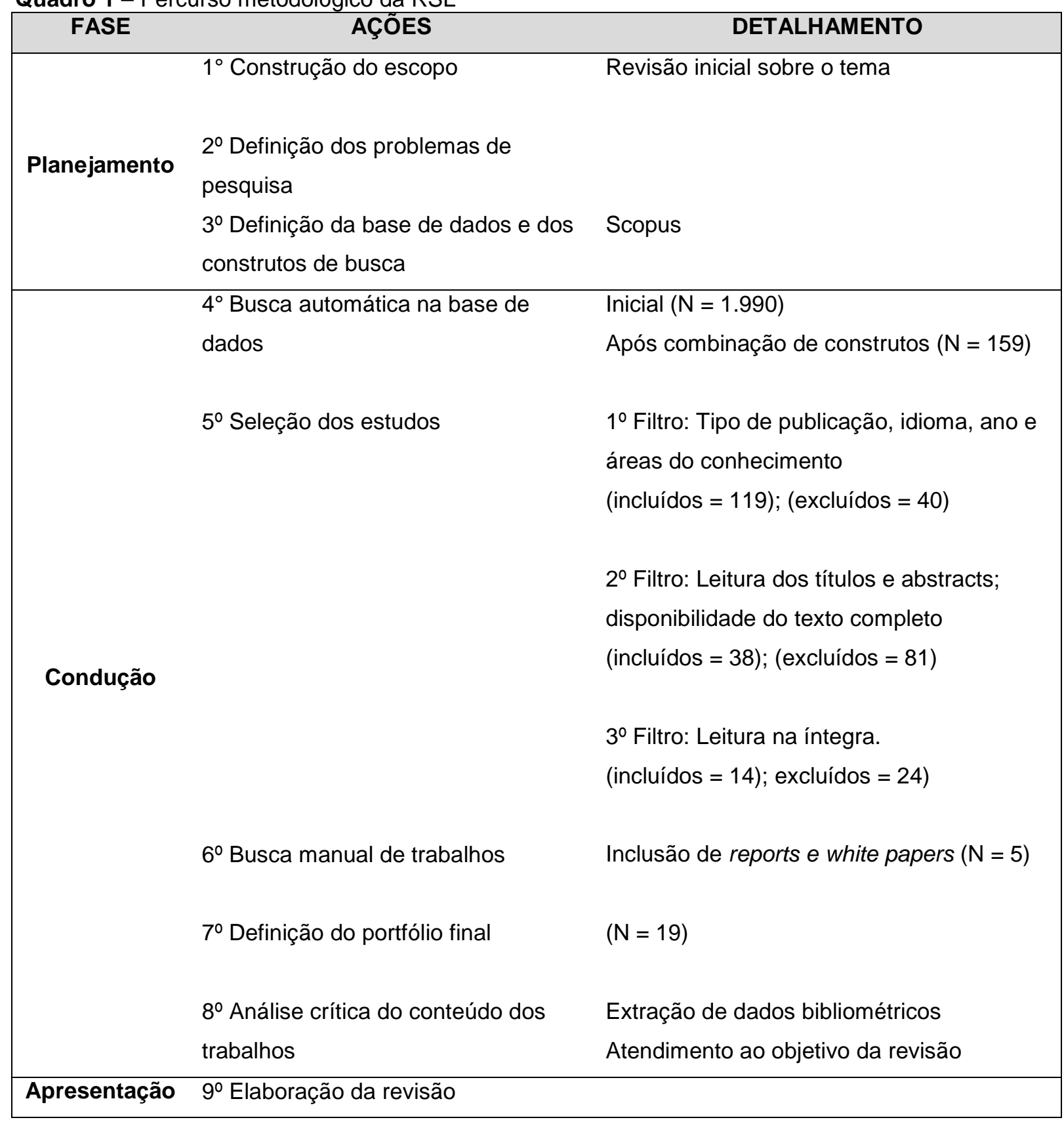

Fonte: Elaborado pelos autores

\section{APRESENTAÇÃO DOS RESULTADOS}

A literatura sobre o tema é escassa, evidenciando a existência de um campo de estudo ainda em construção, com diferentes atores envolvidos na tentativa de contribuir para a expansão de um debate menos centrado na inovação tecnológica que viabiliza a Indústria 4.0 e mais em suas implicações para o trabalho e o trabalhador. 
Inicialmente, acreditamos ser importante apresentar uma visão panorâmica dos trabalhos localizados. Entre os 19 estudos, 10 foram publicados em periódicos, 2 em anais de congressos internacionais, 2 são provenientes de capítulos de livros e há ainda 2 white papers e 3 reports. À primeira vista, o que chama a atenção é que o número de publicações sobre o tema vem crescendo gradativamente: houve 1 publicação em 2014, 3 em 2015, 9 em 2016 e em 2017, até junho, 6 publicações. Depreende-se, com isso, que assim como todas as facetas da Indústria 4.0, a sua associação com o trabalho também se encontra em viés de alta.

Em termos metodológicos, predomina-se nos documentos selecionados, o método de estudo do tipo teórico, o que reflete a urgência de pesquisas empíricas e aplicadas diretamente em campo. Entende-se que o baixo número de surveys ou estudos de caso realizados até o momento ocorrem em função do fato que os efeitos da Indústria 4.0 sobre o trabalho ainda estão no campo da especulação e somente se concretizarão a partir da expansão definitiva do modelo ao redor do mundo.

O quadro 2 apresenta uma síntese de cada trabalho adotado nesta pesquisa:

Quadro 2 - Portfólio de trabalhos selecionados

\begin{tabular}{|c|c|c|c|}
\hline Autor(Es) & $\begin{array}{c}\text { Tipo de } \\
\text { Publicação }\end{array}$ & Método & Objetivo/Enfoque \\
\hline $\begin{array}{c}\text { Becker e } \\
\text { Stern (2016) }\end{array}$ & Periódico & $\begin{array}{l}\text { Estudo } \\
\text { teórico }\end{array}$ & $\begin{array}{l}\text { Apresenta uma estimativa de tendência sobre o } \\
\text { declínio, crescimento ou modificações no emprego com } \\
\text { a predominância dos sistemas ciber-físicos no } \\
\text { ambiente de produção. }\end{array}$ \\
\hline $\begin{array}{l}\text { Benesova e } \\
\text { Tupa (2017) }\end{array}$ & Periódico & $\begin{array}{l}\text { Estudo } \\
\text { teórico }\end{array}$ & $\begin{array}{l}\text { Identifica as qualificações e habilidades requeridas } \\
\text { para diferentes categorias profissionais das áreas de } \\
\text { Tecnologia da Informação e de Produção no âmbito da } \\
\text { Indústria 4.0. }\end{array}$ \\
\hline $\begin{array}{l}\text { Boston } \\
\text { Consulting } \\
\text { Group } \\
(2015 \mathrm{~b})\end{array}$ & Report & Survey & $\begin{array}{l}\text { Impactos da Indústria } 4.0 \text { no mercado de trabalho } \\
\text { alemão até } 2025 \text {. }\end{array}$ \\
\hline $\begin{array}{l}\text { Caruso } \\
(2017)\end{array}$ & Periódico & $\begin{array}{l}\text { Estudo } \\
\text { teórico }\end{array}$ & $\begin{array}{l}\text { Debate os efeitos da Indústria } 4.0 \text { sobre o emprego } \\
\text { através de uma análise histórica das mudanças sociais } \\
\text { provocadas por inovações digitais no sistema } \\
\text { capitalista. }\end{array}$ \\
\hline $\begin{array}{l}\text { Edwards e } \\
\text { Ramirez } \\
\text { (2016) }\end{array}$ & Periódico & $\begin{array}{l}\text { Estudo } \\
\text { teórico }\end{array}$ & $\begin{array}{l}\text { Propõe reflexões sobre o impacto das novas } \\
\text { tecnologias nos trabalhadores a partir de diversos } \\
\text { exemplos ilustrativos. }\end{array}$ \\
\hline Freddi (2017) & Periódico & $\begin{array}{l}\text { Estudo } \\
\text { de } \\
\text { casos } \\
\text { múltiplo } \\
\text { s }\end{array}$ & $\begin{array}{l}\text { Apresenta os resultados do estudo de múltiplos casos } \\
\text { de digitalização do trabalho industrial, atual e futuro, no } \\
\text { âmbito da manufatura Italiana, e os seus possíveis } \\
\text { impactos no emprego. }\end{array}$ \\
\hline $\begin{array}{l}\text { Gehrke et al } \\
\quad(2015)\end{array}$ & White paper & $\begin{array}{l}\text { Diverso } \\
\mathrm{s}\end{array}$ & $\begin{array}{l}\text { Discute a questão do desenvolvimento de habilidades } \\
\text { para o trabalho nas fábricas do futuro. }\end{array}$ \\
\hline
\end{tabular}


Quadro 2 - Portfólio de trabalhos selecionados

(conclusão)

\begin{tabular}{|c|c|c|c|}
\hline & & & \\
\hline Autor(Es) & $\begin{array}{c}\text { Tipo de } \\
\text { Publicação }\end{array}$ & Método & Objetivo/Enfoque \\
\hline $\begin{array}{l}\text { Gorecky et al } \\
(2014)\end{array}$ & Congresso & & $\begin{array}{l}\text { Debate a questão da interação entre o homem e os } \\
\text { sistemas ciber-físicos. }\end{array}$ \\
\hline $\begin{array}{l}\text { Heclkau et al } \\
\qquad(2016)\end{array}$ & Periódico & $\begin{array}{l}\text { Modelag } \\
\text { em }\end{array}$ & $\begin{array}{l}\text { Apresenta um modelo de desenvolvimento de } \\
\text { competências e mostra como as empresas podem } \\
\text { utilizá-lo para enfrentar os desafios surgidos com a } \\
\text { Indústria 4.0. }\end{array}$ \\
\hline $\begin{array}{l}\text { Hirsch- } \\
\text { Kreinsen } \\
(2016)\end{array}$ & Periódico & $\begin{array}{l}\text { Estudo } \\
\text { teórico }\end{array}$ & $\begin{array}{l}\text { Impactos dos sistemas de produção inteligentes no } \\
\text { trabalho industrial. }\end{array}$ \\
\hline $\begin{array}{l}\text { Jasiulewicz- } \\
\text { Kaczmarek et } \\
\text { al (2017) }\end{array}$ & $\begin{array}{l}\text { Capítulo de } \\
\quad \text { livro }\end{array}$ & & $\begin{array}{l}\text { Apresenta os principais desafios que deverão ser } \\
\text { enfrentados pelo trabalhador com o gradativo } \\
\text { crescimento das fábricas inteligentes. }\end{array}$ \\
\hline Peters (2016) & Periódico & $\begin{array}{l}\text { Estudo } \\
\text { teórico }\end{array}$ & $\begin{array}{l}\text { Discute o papel da educação na era do desemprego } \\
\text { causado pela incorporação de novas tecnologias no } \\
\text { trabalho. }\end{array}$ \\
\hline $\begin{array}{l}\text { Romero et al } \\
\text { (2016) }\end{array}$ & $\begin{array}{l}\text { Capitulo de } \\
\text { livro }\end{array}$ & & $\begin{array}{l}\text { Discute o desenvolvimento de sistemas de produção } \\
\text { caracterizados pela automação do trabalho humano. }\end{array}$ \\
\hline $\begin{array}{c}\text { Salento } \\
(2017)\end{array}$ & Periódico & $\begin{array}{l}\text { Estudo } \\
\text { teórico }\end{array}$ & $\begin{array}{l}\text { Propõe, a partir de uma perspectiva sociológica, } \\
\text { reflexões sobre o impacto da digitalização no emprego. }\end{array}$ \\
\hline $\begin{array}{l}\text { Schuh et al } \\
\quad(2015)\end{array}$ & Periódico & & $\begin{array}{l}\text { Propõe uma abordagem integrativa entre as } \\
\text { características e tecnologias da Indústria } 4.0 \text { e a } \\
\text { promoção da aprendizagem baseada no trabalho. }\end{array}$ \\
\hline $\begin{array}{l}\text { Shamim } \\
(2016)\end{array}$ & Congresso & $\begin{array}{l}\text { Estudo } \\
\text { teórico }\end{array}$ & $\begin{array}{l}\text { Propõe práticas de gestão para tornar a organização } \\
\text { compatível com os preceitos da Indústria } 4.0 \text {, por meio } \\
\text { do desenvolvimento de um clima organizacional de } \\
\text { aprendizagem e inovação capaz de melhorar as } \\
\text { capacidades organizacionais. }\end{array}$ \\
\hline Weber (2016) & Report & Survey & $\begin{array}{l}\text { Aborda os principais impactos da Indústria } 4.0 \text { no } \\
\text { mercado de trabalho alemão até } 2030 \text {. }\end{array}$ \\
\hline $\begin{array}{c}\text { World } \\
\text { Economic } \\
\text { Forum (2016) }\end{array}$ & Report & Survey & $\begin{array}{l}\text { Apresenta os resultados de uma pesquisa global sobre } \\
\text { as principais transformações em curso no mundo do } \\
\text { trabalho. }\end{array}$ \\
\hline $\begin{array}{l}\text { World } \\
\text { Economic } \\
\text { Forum (2017) }\end{array}$ & White paper & Survey & $\begin{array}{l}\text { Discute a importância de transformações nos sistemas } \\
\text { educacionais, tanto pelo setor público quanto privado, } \\
\text { visando a preparar as pessoas para o pleno } \\
\text { desenvolvimento de seu potencial. }\end{array}$ \\
\hline
\end{tabular}

Fonte: Elaborado pelos autores

Verificou-se que os 19 trabalhos foram escritos por 52 autores e, como esperado, a maioria (25\%) são alemães, seguido por 7\% de norte-americanos, 7\% italianos e 7\% ingleses. O restante é procedente de inúmeros países, como Austrália, Polônia, Nova Zelândia entre outros. Não há nenhum pesquisador brasileiro na lista. Isso reforça o que já observado inicialmente: a Alemanha, até mesmo por ser o berço da Indústria 4.0, é o país que mais tem se dedicado a pesquisas nesse âmbito. Além disso, dentre o portfólio analisado, cada autor publicou apenas um artigo, o que permite concluir que ainda não há pesquisadores trabalhando preponderantemente com a Indústria 4.0 sob a perspectiva do trabalho. 
Entre os 10 trabalhos publicados em periódicos, o destaque é para as Revistas Al \& Society e Procedia CIRP que publicaram três vezes no período. No quadro 3 apresenta-se um resumo de cada periódico, incluindo seu fator de impacto mais atual (referente a 2016) medido pelo índice SJR (SCImago Journal \& Country Rank), disponibilizado pela base de dados escolhida para esta pesquisa - Scopus - e que em sua avaliação considera, além do número de citações, o prestígio da revista na qual o artigo foi citado.

Quadro 3 - Periódicos que mais publicaram

\begin{tabular}{|l|l|c|c|}
\hline \multicolumn{1}{|c|}{ PERIÓDICO } & \multicolumn{1}{c|}{ ÁREA } & PUBLICAÇÕES & SJR \\
\hline Al \& Society & Interdisciplinar & 3 & 0.175 \\
\hline Procedia CIRP & Engenharias & 3 & - \\
\hline Journal for Labour Market Research & Interdisciplinar & 1 & 0.371 \\
\hline New Technology, Work and Employment & Interdisciplinar & 1 & 0.653 \\
\hline Procedia Manufacturing & Engenharias & 1 & 0.105 \\
\hline Educational Philosophy and Theory & Educação & 1 & 0.418 \\
\hline
\end{tabular}

Fonte: Elaborado pelos autores

Analisamos também os trabalhos que receberam o maior número de citações. Era previsto, e assim foi confirmado, que as pesquisas houvessem recebido poucas citações até o momento em função da incipiência do tema. O único trabalho que se destaca nesse quesito é justamente o mais antigo deste portfólio. Trata-se do artigo "Human-Machine-Interaction in the Industry 4.0 Era" publicado em 2014 no 12th IEEE International Conference on Industrial Informatics e cujo autores estão vinculados ao conceituado German Research Center for Artificial Intelligence, um dos maiores institutos de pesquisa em inteligência artificial do mundo.

Quadro 4 - Publicações mais citadas

\begin{tabular}{|l|l|l|c|}
\hline ANO & \multicolumn{1}{|c|}{ AUTOR(ES) } & \multicolumn{1}{c|}{ TíTULO } & CITAÇÕES \\
\hline 2014 & Gorecky et al & Human-Machine-Interaction in the Industry 4.0 Era & 54 \\
\hline 2015 & Schuh et al & Promoting work-based learning through Industry 4.0 & 9 \\
\hline 2016 & Heclkau et al & $\begin{array}{l}\text { Holistic approach for human resource management in } \\
\text { Industry 4.0 }\end{array}$ & 4 \\
\hline 2017 & $\begin{array}{l}\text { Jasiulewicz- } \\
\text { Kaczmarek et al }\end{array}$ & $\begin{array}{l}\text { The maintenance management in the macro- } \\
\text { ergonomics context }\end{array}$ & 4 \\
\hline 2016 & Hirsch-Kreinsen & $\begin{array}{l}\text { Digitization of industrial work: development paths and } \\
\text { prospects }\end{array}$ & 3 \\
\hline 2016 & Shamim & $\begin{array}{l}\text { The Operator 4.0: Human Cyber-Physical Systems \& } \\
\text { Adaptive Automation towards Human-Automation } \\
\text { Symbiosis Work Systems }\end{array}$ & 3 \\
\hline
\end{tabular}

Fonte: Elaborado pelos autores 


\section{DISCUSSÃO E ANÁLISE DE CONTEÚDO}

Os trabalhos foram exaustivamente lidos e, após a análise de conteúdo, foi possível identificar e categorizar quatro principais impactos provocados pela Indústria 4.0 na organização do trabalho (quadro 5). Nota-se que alguns estudos, dado seu escopo e abrangência, abordam mais de um impacto, enquanto outros centralizam sua pesquisa em uma questão mais particular. Há que salientar ainda, que, embora distintos, todos os impactos são inter-relacionados e interdependentes.

Quadro 5 - Impactos da Indústria 4.0 na organização do trabalho

\begin{tabular}{|c|c|}
\hline IMPACTO & FONTE \\
\hline Aumento do desemprego tecnológico, em & BCG (2015b) \\
contrapartida a criação e/ou aumento de & Becker e Stern (2016) \\
postos de trabalho mais complexos e & Edwards e Ramirez (2016) \\
qualificados & Freddi (2017) \\
& Peters (2016) \\
& Salento (2017) \\
& Weber (2016) \\
WEF (2016) \\
Necessidade de desenvolvimento de novas & BCG (2015b) \\
competências e habilidades & Benesova e Tupa (2017) \\
& Edwards e Ramirez (2016) \\
& Gehrke et al (2015) \\
& Heclkau et al (2016) \\
Maior interação entre o homem e a máquina & Jasiulewicz-kaczmarek et al (2017) \\
& Schuh et al (2015) \\
& Weber (2016) \\
Transformações nas relações & WEF (2016; 2017) \\
socioprofissionais & BCG (2015b) \\
& Romero et al (2016) \\
& Caruso (2017) \\
& Edwards e Ramirez (2016) \\
& Gorecky et al (2014) \\
& Hirsch-Kreinsen (2016) \\
& Jasiulewicz-kaczmarek et al (2017) \\
& Shamim (2016) \\
& WEF (2016) \\
\hline
\end{tabular}

Fonte: Elaborado pelos autores

\subsection{Postos de Trabalho}

O impacto no número de empregos é o mais controverso e polêmico efeito da Indústria 4.0. Os debates têm sido polarizados por aqueles que acreditam em oportunidades ilimitadas de novos empregos e aqueles que preveem maciça substituição da mão de obra e o desaparecimento de postos de trabalho (WEF, 2016). 
Essa controvérsia fica explicita quando analisados dois estudos com objetivos semelhantes: o do Boston Consulting Group (2015b) e o do Institute for Employment Research, realizado por Weber (2016). Ambos se propõem a esmiuçar o impacto da Indústria 4.0 no mercado de trabalho alemão, com a diferença de que o primeiro tem como parâmetro o horizonte do ano de 2025 e o segundo, o ano de 2030. Para o BCG (2015b) a questão do emprego está diretamente relacionada a duas variáveis: o crescimento de receita gerado pelas novas tecnologias e o percentual de adoção destas pelas empresas. Após a análise de vários possíveis cenários, a pesquisa conclui que o mais provável é que a Indústria 4.0 leve a um aumento de 350 mil empregos na Alemanha. Por outro lado, Weber (2016) prevê 60 mil vagas a menos no país.

Em 2015, o World Economic Forum realizou a maior pesquisa global sobre o assunto, cujo objetivo principal era compreender como as mudanças tecnológicas e sociodemográficas em andamento afetariam o trabalho até 2020. Foram entrevistados CEO's (Chief Executiver Officer), CHRH's (Chief Human Resources Officer) e outros executivos de alto escalação de 371 empresas, de 9 diferentes setores industriais, espalhadas nos 15 países ou áreas econômicas mais desenvolvidos ou emergentes (incluindo o Brasil). Somadas, essas empresas possuem mais de 13 milhões de empregados e os países onde estão localizadas concentram $65 \%$ da força de trabalho mundial (aproximadamente 1,86 bilhões).

Os resultados da pesquisa foram divulgados em 2016 no Report "The Futere of Jobs: Employment, Skills and Workforce Strategy for the Fourth Industrial Revolution" e apontam que a quarta revolução industrial provocará a perda de 7,1 milhões de empregos, enquanto 2 milhões serão criados, resultando em um impacto negativo de 5,1 milhões de postos de trabalho até 2020 , dos quais somente no setor de manufatura e produção mais de 1,6 milhões de empregos diretos serão substituídos por robôs e outras tecnologias avançadas.

Não parece exagero, portanto, falar que Indústria 4.0 pode ser a grande responsável por alimentar o fenômeno do desemprego tecnológico, que é aquele causado pelo uso massivo de tecnologias tornando obsoleto o trabalho humano. Tal fenômeno é, sem dúvida, um problema iminente que criará maiores desigualdades e um abismo entre os retornos ao trabalho e o retorno ao capital (PETERS, 2016; SALENTO; 2017). Contudo, assim como observado por Salento (2017), as inovações 
tecnológicas não criam, sozinhas, mercados ou prosperidade, nem favorecem o desemprego ou a desigualdade. O que está em jogo são as escolhas políticas, regulatórias e sociais dos governos, empresas e da sociedade em geral. Para o autor, muito mais do que uma revolução industrial, a Indústria 4.0 pode ser encarada como um programa transnacional de reindustrialização, conduzido por coalizões de grandes corporações e governos nacionais, com o objetivo de reestruturar a rentabilidade do capital industrial, em grande parte ultrapassada nos últimos anos por investimentos financeiros.

Não obstante as controvérsias numéricas, o que parece ser consenso é que a criação de novas vagas dar-se-á em níveis gerenciais ou em áreas que exigem maior qualificação, como ciências matemáticas e da computação, engenharia e arquitetura; enquanto o declínio de empregos ocorrerá principalmente em tarefas simples e rotineiras e portanto mais suscetíveis à automação (BCG, 2015b; WEF, 2016; WEBER, 2016; BECKER; STERN, 2016).

Essa questão pode ser melhor compreendida a partir da pesquisa realizada pelo BCG (2015b) onde é possível identificar algumas transformações geradas pela tecnologia no ambiente de produção e como elas afetarão a redução e a criação de empregos em determinadas áreas de atuação:

\begin{tabular}{|c|c|c|}
\hline TRANSFORMAÇÃO & REDUÇÃO DE EMPREGOS & CRIAÇÃO DE EMPREGOS \\
\hline $\begin{array}{l}\text { Utilização do Big Data no } \\
\text { controle de qualidade }\end{array}$ & $\begin{array}{l}\text { Especialistas em controle } \\
\text { de qualidade }\end{array}$ & $\begin{array}{l}\text { - } \quad \text { Analistas de dados } \\
\text { industriais }\end{array}$ \\
\hline $\begin{array}{c}\text { Utilização de robôs, } \\
\text { veículos autônomos e } \\
\text { impressoras 3D nas linhas } \\
\text { de produção }\end{array}$ & $\begin{array}{l}\text { - Operadores de produção, } \\
\text { montagem e embalagem } \\
\text { - } \quad \text { Pessoal de logística }\end{array}$ & $\begin{array}{ll}\text { - } & \text { Coordenadores de robôs } \\
\text { - } & \text { Engenheiros e } \\
\text { especialistas em pesquisa } \\
\text { e desenvolvimento }\end{array}$ \\
\hline $\begin{array}{l}\text { Redes de suprimentos e } \\
\text { linhas de produção } \\
\text { autônomas e inteligentes }\end{array}$ & $\begin{array}{l}\text { - Especialistas em } \\
\text { planejamento de produção }\end{array}$ & $\begin{array}{l}\text { Especialistas em } \\
\text { modelagem e } \\
\text { interpretação de dados }\end{array}$ \\
\hline $\begin{array}{c}\text { Manutenção preditiva } \\
\text { automatizada }\end{array}$ & $\begin{array}{l}\text { - Técnicos de manutenção } \\
\text { tradicionais }\end{array}$ & $\begin{array}{l}\text { Analistas de dados, } \\
\text { sistemas e } \mathrm{TI}\end{array}$ \\
\hline
\end{tabular}

Fonte: Elaborado pelos autores a partir do BGC (2015b)

Nesse mesmo sentido, Freddi (2017), em um estudo com diversas indústrias italianas, analisa como essas corporações estão planejando e lidando com seus futuros processos inovativos através da incorporação das principais tecnologias associadas à Indústria 4.0, como Big Data, IoT e manufatura aditiva. O estudo conclui que é esperado que essas inovações levem a efeitos positivos sobre o emprego, 
sobretudo na expansão de serviços ao consumidor e nas áreas de softwares e análise de dados, nas quais as empresas apontam que já existe atualmente bastante dificuldade em encontrar profissionais qualificados.

\subsection{Desenvolvimento de competências}

A necessidade do aperfeiçoamento de competências e habilidades é uma consequência lógica da geração de empregos que exigem uma maior qualificação. Apropriando-se do termo utilizado por Edwards e Ramirez (2016), trata-se de uma "reciclagem" do trabalhador, na qual, semelhante às três primeiras revoluções industriais, esta quarta exige a adaptação às novas tecnologias e às mudanças organizacionais que elas provocam, com vistas a manter as condições de empregabilidade.

$\mathrm{Na}$ literatura, manifestam-se diferentes abordagens e metodologias utilizadas pelos autores para detectar e qualificar as competências requeridas pela Indústria 4.0 e pelas Smart Factories, entretanto, todas acabam apresentando uma série de competências e habilidades comuns que, progressivamente, serão essenciais aos trabalhadores, independentemente da função exercida e de suas peculiaridades.

Hecklau et al (2016) apresentam um modelo de desenvolvimento de competências baseado nos desafios impostos à Indústria 4.0 - já descritos neste trabalho -, identificando quais competências serão necessárias para superá-los. Benesova e Tupa (2017) entendem que os profissionais de nível superior nas áreas de tecnologia da informação e de produção serão os principais beneficiados pela Indústria 4.0, contudo, os autores listam inúmeras qualificações acadêmicas e habilidades em geral que tais profissionais deverão possuir. Gehrke et al (2015) e o World Economic Forum (2016) apresentam diversas competências, divididas em várias categorias, ranqueando-as conforme sua relevância. Outros estudos, como o de Jasiulewicz-Kaczmarek et al (2017), de Schuh et al (2015), de Weber (2016) e do BCG (2015b) também citam algumas competências, embora não seja o foco de suas respectivas pesquisas.

Visando sintetizar e compilar essas diferentes perspectivas, identificamos as principais competências comuns às pesquisas e classificamo-las em três categorias, sem qualquer tipo de predominância entre uma e outra: (1) Competências funcionais 
- entendidas como aquelas necessárias para o desempenho técnico e profissional das tarefas; (2) Competências comportamentais - mais intrínsecas e relacionadas às atitudes do indivíduo; e (3) Competências sociais - relacionadas com a capacidade de interagir e trabalhar com outras pessoas. O quadro 7 apresenta o resultado desse trabalho:

\begin{tabular}{|c|c|}
\hline \multirow{6}{*}{$\begin{array}{l}\text { Competências } \\
\text { funcionais }\end{array}$} & Resolução de problemas complexos \\
\hline & Conhecimento avançados em TI, incluindo codificação e programação \\
\hline & Capacidade de processar, analisar e proteger dados e informações \\
\hline & Operação e controle de equipamentos e sistemas \\
\hline & Conhecimento estatístico e matemático \\
\hline & Alta compreensão dos processos e atividades de manufatura \\
\hline \multirow{6}{*}{$\begin{array}{l}\text { Competências } \\
\text { comportamentais }\end{array}$} & Flexibilidade \\
\hline & Criatividade \\
\hline & Capacidade de julgar e tomar decisões \\
\hline & Autogerenciamento do tempo \\
\hline & Inteligência emocional \\
\hline & Mentalidade orientada para aprendizagem \\
\hline \multirow{6}{*}{$\begin{array}{l}\text { Competências } \\
\text { sociais }\end{array}$} & Habilidade de trabalhar em equipe \\
\hline & Habilidades de comunicação \\
\hline & Liderança \\
\hline & Capacidade de transferir conhecimento \\
\hline & Capacidade de persuasão \\
\hline & Capacidade de comunicar-se em diferentes idiomas \\
\hline
\end{tabular}

Fonte: Elaborado pelos autores a partir de BCG (2015b), Gehrke et al (2015), Schuh et al (2015), Heclkau et al (2016), Weber (2016), WEF (2016), Jasiulewicz-kaczmarek et al (2017) e Benesova e Tupa (2017)

Depreende-se dessa análise que as competências identificadas, associadas ao advento da Indústria 4.0, não são, necessariamente, novas habilidades. O que muda com sua expansão é a maior exigência dessas competências e o reconhecimento explícito de que os trabalhadores que não as possuírem fatalmente perderão seu emprego (EDWARDS; RAMIREZ, 2016). Entende-se a partir disso que os trabalhadores das fábricas do futuro serão muito mais generalistas do que especialistas, devendo possuir conhecimentos interdisciplinares sobre a organização, os processos e as tecnologias (GEHRKE et al, 2015).

O ponto central da discussão, então, passa a ser como desenvolver essas competências de forma a promover o potencial humano nas organizações e atingir aos anseios desta quarta revolução. Duas principais estratégias emergem como urgentes: a primeira, relacionada à aprendizagem e à inovação no ambiente de trabalho; e a segunda, uma necessidade de reformulação nos sistemas educacionais, unificando os interesses públicos, privados e científicos. 
O dever das corporações buscarem a atualização das competências e habilidades de sua força de trabalho é ponto pacífico entre os autores. Em seu trabalho, Schuh et al (2015) analisam especificamente essa questão, desenvolvendo um modelo teórico-prático para a promoção da aprendizagem baseada do trabalho. Tal modelo, passível de ser replicado em diversas empresas, combina as características da Indústria 4.0 com algumas "alavancas" centrais relacionadas à organização e às técnicas de trabalho, buscando, dessa forma, reduzir a complexidade do aprendizado, aumentar a motivação para aprender e apoiar o trabalhador durante o processo de aprendizagem. O WEF (2016) também aborda a questão da importância do aprendizado, mas defende que este deve ocorrer de forma constante, por toda a vida e não somente no ambiente laboral, uma vez que não há a possiblidade de adquirir todas as habilidades exigidas somente através do trabalho.

Em relação à segunda estratégia, a de reformulação dos sistemas educacionais, percebe-se que não há uma definição concreta do que seja exatamente essa reformulação, mas é consenso que ela deve estar em sintonia com a nova revolução industrial em curso.

As pesquisas realizadas pelo BCG (2015b), pelo WEF (2017), por Weber (2016) e por Gehrke et al (2015) indicam diversas ações para orientar a formulação de novas políticas educacionais onde a tecnologia da informação seja incorporada em todos os níveis de ensino, da pré-escola ao ensino superior. Entre elas, destacam-se: (1) qualificar a força de trabalho docente para que estejam aptos a aplicar distintas tecnologias no processo de ensino-aprendizagem; (2) adaptar os currículos dos cursos superiores para ofertar maiores conhecimentos interdisciplinares em TI, engenharia, matemática, comunicação e administração, buscando preencher as lacunas de competências identificadas pela Indústria 4.0; e (3) fortalecer o ensino técnico e profissional, voltado para a realidade do mercado de trabalho local.

\subsection{Interação homem-máquina}

O terceiro impacto a ser provocado pela Indústria 4.0 no trabalho é a maior interação entre o homem e a máquina, trazendo, segundo o BCG (2015b), significativas mudanças para a natureza do trabalho industrial e para a estrutura organizacional das firmas. 
Esse novo modelo de interação é viabilizado pelo surgimento dos CyberPhysical Systems, os quais possibilitam a construção de uma nova forma de organização do trabalho caracterizada pela integração entre o homem, a máquina e a tecnologia. Segundo Romero et al (2016) ela aposta em diversas ferramentas e tecnologias que permitem ao homem "dialogar" com a máquina, principalmente pelo uso de dispositivos móveis como smartphones, tablets e smartglasses, capazes de permitir controles operacionais por meio de telas sensíveis ao toque, reconhecimento de voz e reconhecimento de gestos, tornando o trabalho mais intuitivo, imediato e eficiente.

O BCG (2015b) lista alguns exemplos onde essa maior interação proporcionará benefícios ao trabalhador e à organização, destacando-se:

- Produção assistida por robôs: flexíveis e humanoides robôs podem ajudar a mitigar os efeitos físicos de tarefas repetitivas e desgastantes, proporcionando um trabalho ergonomicamente mais correto, além de ser útil em países com a força de trabalho mais idosa, permitindo assim que as pessoas nessas condições possam continuar trabalhando por mais tempo de forma segura e saudável;

- Manutenção preditiva: o trabalho dos técnicos de manutenção de máquinas será essencialmente preditivo e o serviço poderá ocorrer de forma remota. Essa produtividade reduzirá drasticamente o tempo de inatividade da máquina e o tempo levado pelo profissional em sua manutenção;

- Controle operacional: os avanços da Indústria 4.0 possibilitarão que um operador se responsabilize por várias máquinas. Medidas de operação padrão para qualquer tipo de tarefas e dados serão mostradas em tempo real em telas e óculos, permitindo que o monitoramento da qualidade da performance do produto e da máquina seja realizado por meio de consultas em um sistema automatizado. Exigir-se-á, assim, menos treinamento específico sobre o funcionamento das máquinas e mais na capacidade de utilizar dispositivos digitais e softwares para acessar um repositório de conhecimento virtual.

Ainda sob essa perspectiva, Romero et al (2016), em analogia ao termo principal, apresentam o conceito de "Operário 4.0": um trabalhador inteligente e 
habilidoso que executa o trabalho auxiliado pelas máquinas e que pode até mesmo configurar-se em uma nova filosofia de engenharia de produção na qual a automação é vista como um aprimoramento das capacidades físicas, sensoriais e cognitivas do homem.

\subsection{Relações socioprofissionais}

Decorrentes de todos os outros impactos observam-se alguns efeitos indiretos provocados pela Indústria 4.0, os quais denominamos de transformações nas relações socioprofissionais, abrangendo aspectos do cotidiano de gestão e organização da força de trabalho que inevitavelmente serão afetados pela maior digitalização da produção.

Caruso (2017) analisa particularmente essa questão e conclui que, igualmente às revoluções industriais anteriores, na Indústria 4.0, a organização do trabalho não se tornará mais horizontal, e diferentemente do prometido, o poder de decisão e a autonomia dos trabalhadores não aumentará. Para o autor, que apresenta uma visão bastante crítica, a Indústria 4.0 intensificará os já conhecidos resultados concretos das transformações dos processos produtivos ocorridos sob a égide do sistema capitalista: redução da força de trabalho, redução dos direitos e garantias dos trabalhadores e o aumento da concentração de capital e do monopólio das forças de produção.

A possibilidade de precarização das relações de trabalho também é observada pelo WEF (2016) e por Edwards e Ramirez (2016) que afirmam que provavelmente a Indústria 4.0 levará à expansão de trabalhadores independentes contratados para a realização de serviços pré-determinados através de plataformas digitais, por um curto período de tempo, e em um contexto de insegurança e ausência de benefícios.

Aspectos como liderança e práticas cotidianas de gestão de pessoas também devem ser repensadas para o alinhamento ao modelo 4.0. Shamim et al (2016) entendem que o estilo de liderança transformacional (caracterizado por estímulos à motivação e à inspiração individual como forma de proporcionar mudanças organizacionais) combinado com uma visão de liderança orientada para o conhecimento é o modelo mais indicado para atender às pressuposições da Indústria 4.0. Nessa mesma linha, o WEF (2016) aponta para a inevitável reinvenção da função de gestão de pessoas, a qual deve empregar novos tipos de ferramentas analíticas 
para detectar talentos e lacunas de habilidades, fornecendo informações que ajudem as organizações a alinhar suas estratégias de negócios, gestão e inovação.

Além disso, partindo do pressuposto da maior interação entre o homem e a máquina, Gorecky (2014), entende que isso leve a um debate sócio-tecnológico sobre autonomia e poder de decisão do tipo: Quem faz o quê? Quem controla quem? HirschKreinsen (2016) identifica duas correntes de pensamento sobre o tema: a primeira pertencente a um conceito de automação centrada na tecnologia, onde as máquinas assumem a responsabilidade pelo trabalho, restando ao homem apenas tarefas compensatórias, que não podem ou não são viáveis de serem automatizadas. Já a segunda, está relacionada a um conceito de automação complementar, com tarefas distribuídas entre o homem e a máquina, em uma perspectiva colaborativa e sinérgica para o pleno desempenho do sistema.

Os autores apresentados nesta pesquisa parecem concordar com a segunda visão, em que se evidencia, inclusive, a existência de um discurso carregado de romantismo, onde o trabalhador é visto como elemento indispensável para o sucesso da Indústria 4.0 (JASIULEWICZ-KACZMAREK et al, 2017) e como a parte mais flexível do processo, cuja responsabilidade deve ser a de utilizar suas competências, conhecimentos e capacidade de adaptação para gerenciar as máquinas e os sistemas, denotando sua superioridade (GORECKY, 2014).

\section{CONCLUSÕES}

Há que se reconhecer que, tanto no meio industrial quanto acadêmico, a Indústria 4.0 esteja em sua mais tenra idade, apesar dos inúmeros esforços de marketing tentando mostrar o contrário (DRATH, HORCH, 2014). Em termos acadêmicos, verifica-se um amplo campo de pesquisa ainda em construção, sendo abordado por diferentes áreas do conhecimento através de diversas perspectivas. No Brasil, o debate encontra-se em fase ainda mais inicial, com poucas e pontuais pesquisas sobre o tema, com destaque para reuniões científicas como o ENEGEP Encontro Nacional de Engenharia de Produção, que abordou o assunto em sua edição de 2017.

Dentre todos os possíveis impactos da Indústria 4.0, esta pesquisa limitou-se a analisar aqueles diretamente relacionados ao trabalho. Empregando o método de 
revisão sistemática da literatura, construímos inicialmente uma visão geral sobre a produção acadêmica na área, onde concluímos que, assim como os demais, os impactos relacionados ao trabalho ainda se encontram no campo da especulação e das contradições. Verifica-se também que, até então, não há pesquisadores atuando majoritariamente com a Indústria 4.0 sob a perspectiva do trabalho, embora as pesquisas estejam em viés de crescimento, o que nos leva a crer que com a provável expansão do modelo, novos e mais relevantes estudos devem ser efetivados e publicados.

Identificamos, categorizamos e discutimos quatro principais possíveis impactos que nos permitem concluir que a Indústria 4.0 tem potencial para provocar drásticas transformações na natureza do trabalho industrial e caminhar em direção à construção de uma nova forma de organização do trabalho pautada pela união do homem, da máquina e da tecnologia, embora ainda persistam dúvidas acerca de seu caráter revolucionário no sentido de uma maior inclusão social, expressadas, por exemplo, nas possiblidades de eliminação de milhares de postos de trabalho (WEF, 2016; WEBER, 2016) e de precarização das relações socioprofissionais (WEF, 2016; EDWARDS; RAMIREZ, 2016; CARUSO, 2017)

Destarte, as principais contribuições desta pesquisa ocorreram no sentido de apresentar esses impactos; de elencar as principais competências exigidas pelo mercado com a ascensão do modelo 4.0 e de fomentar uma discussão sobre um relevante tema de pesquisa que ainda não recebe a devida atenção dos pesquisadores no Brasil. Já suas limitações resultam de seu escopo e metodologia. Como somente foram analisados publicações em inglês e português, contribuições relevantes em outros idiomas, principalmente no alemão, podem ter sido perdidas. Além disso, durante a definição dos descritores utilizados na busca de estudos, alguns construtos podem ter sido despercebidos, levando a uma seleção incompleta. Aspectos subjetivos de cunho avaliativo realizado pelos pesquisadores também são efeitos limitantes que devem ser considerados.

Como continuidade, para futuras pesquisas sugere-se (1) aprofundar a investigação em qualquer um dos quatro impactos identificados; (2) a condução de pesquisas empíricas, por meio de entrevistas com gestores brasileiros, visando a identificar se estes possuem visões semelhantes ou distintas àquelas apresentadas neste estudo; e (3) a realização de pesquisas onde os impactos no trabalho sejam 
contrapostos a outros impactos, como políticos e econômicos, de forma a obter um debate mais rico e amplo sobre a temática.

Conclui-se, portanto, que embora apresentado neste artigo que a Indústria 4.0 não se trata essencialmente da completa substituição do homem pela tecnologia, parece bastante coerente à posição de Buhr (2017) para o qual a Indústria 4.0 tem que provar seus benefícios para a sociedade e somente quando suas tecnologias, regulamentos e mudanças revelarem-se melhores para as pessoas, é que se deve reconhecer todo o seu potencial.

\section{REFERÊNCIAS}

BAUER, Wilhelm et al. Transforming to a hyper-connected society and economy - towards an "industry 4.0". Procedia Manufacturing, v. 3, p. 417-424, 2015.

DOI: https://doi.org/10.1016/j.promfg.2015.07.200

BECKER, Till; STERN, Hendrik. Future Trends in Human Work area Design for CyberPhysical Production Systems. Procedia CIRP, v. 57, p. 404-409, 2016.

DOI: https://doi.org/10.1016/j.procir.2016.11.070

BENEŠOVÁ, Andrea; TUPA, Jiří. Requirements for Education and Qualification of People in Industry 4.0. Procedia Manufacturing, v. 11, p. 2195-2202, 2017.

DOI: https://doi.org/10.1016/j.promfg.2017.07.366

BOSTON CONSULTING GROUP. Industry 4.0: The future of productivity and growth in manufacturing industries. BCG Perspectives, 2015a. Disponível em: < https://www.bcgperspectives.com/content/articles/engineered products project business in dustry 40 future productivity growth manufacturing industries/ > Acesso em 13 maio 2017.

Man and Machine in Industry 4.0: how will technology transform the industrial workforce through 2025? BCG Perspectives ,2015b. Disponível em: < https://www.bcgperspectives.com/content/articles/technology-business-transformationengineered-products-infrastructure-man-machine-industry-4/ > Acesso em 15 maio 2017.

BUHR, Daniel. Social innovation policy for Industry 4.0. Friedrich-Ebert-Stiftung, Division for Social and Economic Policies, 2015. Disponível em: < http://library.fes.de/pdffiles/wiso/11479.pdf > Acesso em 13 maio 2017.

CARUSO, Loris. Digital innovation and the fourth industrial revolution: epochal social changes? AI \& SOCIETY, p. 1-14, 2017. DOI: https://doi.org/10.1007/s00146-017-0736-1

CONFEDERAÇÃO NACIONAL DA INDÚSTRIA. Desafios para a Indústria 4.0 no Brasil. Brasília, 2016. Disponível em: < http://www.portaldaindustria.com.br/publicacoes/2016/8/desafios-para-industria-40-nobrasil/\# > Acesso em 07 maio 2017.

DAVIES, Ron. Industry 4.0: Digitalisation for productivity and growth. European 
Parliamentary Research Service. Briefing. 2015. Disponível em: < http://www.europarl.europa.eu/RegData/etudes/BRIE/2015/568337/EPRS BRI(2015)568337 EN.pdf > Acesso em 10 maio 2017.

DIEHL, Astor. Antônio; TATIM, Denise Carvalho. Pesquisa em ciências sociais aplicadas: métodos e técnicas. São Paulo: Pearson, 2004.

DRATH, Rainer.; HORCH, Alexander. Industrie 4.0: Hit or hype? IEEE Industrial Electronics Magazine, v. 8, n. 2, p. 56-58, 2014. https://doi.org/10.1109/MIE.2014.2312079

FREDDI, Daniela. Digitalisation and employment in manufacturing. Al \& SOCIETY, p. 1-11, 2017. DOI: https://doi.org/10.1007/s00146-017-0740-5

EDWARDS, Paul; RAMIREZ, Paulina. When should workers embrace or resist new technology? New technology, work and employment, v. 31, n. 2, p. 99-113, 2016. DOI: https://doi.org/10.1111/ntwe.12067

FEDERAÇÃO DAS INDÚSTRIAS DO ESTADO DO RIO DE JANEIRO - FIRJAN. Indústria 4.0. Caderno Senai de Inovação. Rio de Janeiro, 2016. Disponível em: <

http://www.firjan.com.br/publicacoes/publicacoes-de-inovacao/industria-4-0-1.htm > Acesso em 07 maio 2017.

GEHRKE, Lars et al. A Discussion of Qualifications and Skills in the Factory of the Future: A German and American Perspective. VDI/ASME Industry 4.0 White Paper, p. 128, 2015. Disponível em: < http://www.vdi.eu/fileadmin/vdi de/redakteur/karriere bilder/VDIASME 2015 White Paper final.pdf > Acesso em 23 maio 2017.

GREENHALGH, Trisha. How to read a paper: Papers that summarise other papers: systematic reviews and meta-analysis. British Medical Journal, v. 315, p. 672-5, 1997. DOI: https://doi.org/10.1136/bmj.315.7109.672

GORECKY, Dominic et al. Human-machine-interaction in the industry 4.0 era. In: 12th IEEE International Conference on Industrial Informatics. p. 289-294, 2014.

DOI: https://doi.org/10.1109/INDIN.2014.6945523

HECKLAU, Fabian et al. Holistic approach for human resource management in Industry 4.0. Procedia CIRP, v. 54, p. 1-6, 2016. DOI: https://doi.org/10.1016/j.procir.2016.05.102

HERMANN, Mario; PENTEK, Tobias; OTTO, Boris. Design Principles for Industrie 4.0 Scenarios: A Literature Review. Working paper, 2015.

DOI: https://doi.org/10.1109/HICSS.2016.488

HIRSCH-KREINSEN, Hartmut. Digitization of industrial work: development paths and prospects. Journal for Labour Market Research, v. 49, n. 1, p. 1-14, 2016.

DOI: https://doi.org/10.1007/s12651-016-0200-6

JASIULEWICZ-KACZMAREK, Malgorzata et al. The Maintenance Management in the Macro-Ergonomics Context. In: Advances in Social \& Occupational Ergonomics. Springer International Publishing, 2017. p. 35-46. DOI: https://doi.org/10.1007/978-3-319-41688-5 4

KAGERMANN, Henning; WAHLSTER, Wolfgang.; HELBIG, Johannes. Recommendations for implementing the strategic initiative Industrie 4.0. Final Report, Acatech, 2013. Disponível em: < http://www.acatech.de/de/publikationen/stellungnahmen/kooperationen/detail/artikel/recomm 
endations-for-implementing-the-strategic-initiative-industrie-40-final-report-of-the-industr.html $>$ Acesso em 07 maio 2017.

KITCHENHAM, Barbara; CHARTERS, Stuart. Guidelines for performing systematic literature reviews in software engineering. In: Technical report, Ver. 2.3 EBSE Technical Report. EBSE, 2007. Disponivel em <

https://www.elsevier.com/ data/promis misc/525444systematicreviewsquide.pdf $>$ Acesso 18 maio 2017.

PETERS, Michael A. Technological unemployment: Educating for the fourth industrial revolution. Educational Philosophy and Theory, v. 49, n. 1, 2016.

DOI: https://doi.org/10.1080/00131857.2016.1177412

POOVENDRAN, Radha. Cyber-physical systems: Close encounters between two parallel worlds. Proceedings of the IEEE, v. 98, n. 8, p. 1363-1366, 2010.

https://doi.org/10.1109/JPROC.2010.2050377

ROBLEK, Vasja; MESKO, Maja; KRAPEZ, Alojz. A complex view of industry 4.0. SAGE

Open, v. 6, n. 2, p. 1-11, 2016. DOI: https://doi.org/10.1177/2158244016653987

ROMERO, David et al. The Operator 4.0: Human Cyber-Physical Systems \& Adaptive Automation towards Human-Automation Symbiosis Work Systems. In: APMS (Advances in Production Management Systems). 2016.

DOI: https://doi.org/10.1007/978-3-319-51133-7 80

SALENTO, Angelo. Digitalisation and the regulation of work: theoretical issues and normative challenges. Al \& SOCIETY, p. 1-10.

DOI: https://doi.org/10.1007/s00146-017-0738-z

SCHUH, Günther et al. Promoting work-based learning through industry 4.0. Procedia CIRP, v. 32, p. 82-87, 2015. DOI:https://doi.org/10.1016/j.procir.2015.02.213

SCHWAB, Klaus. The Fourth Industrial Revolution. Genebra: World Economic Forum, 2016.

SHAMIM, Saqib et al. Management approaches for Industry 4.0: A human resource management perspective. In: Evolutionary Computation (CEC), 2016 IEEE Congress on. IEEE, 2016. p. 5309-5316. DOI:https://doi.org/10.1109/CEC.2016.7748365

WORLD ECONOMIC FORUM. The future of jobs: Employment, skills and workforce strategy for the fourth industrial revolution. Report. Genebra, 2016. Disponível em: < http://reports.weforum.org/future-of-jobs-2016/ > Acesso em 14 maio 2017.

Realizing Human Potential in the Fourth Industrial Revolution: An Agenda for Leaders to Shape the Future of Education, Gender and Work. White Paper. Genebra, 2017. Disponível em: < https://www.weforum.org/whitepapers/realizing-human-potential-inthe-fourth-industrial-revolution/ . Acesso em 17 maio 2017.

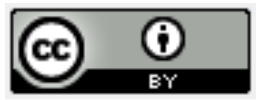

Artigo recebido em 07/07/2017 e aceito para publicação em 01/12/2017

DOI: http://dx.doi.org/10.14488/1676-1901.v18i2.2967 